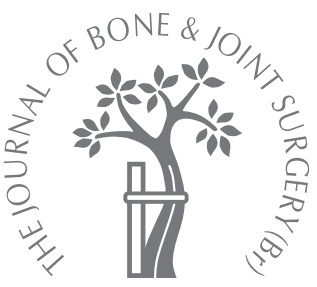

\title{
Treatment of late-stage Freiburg's disease using a temporary metal interpositional device
}

\section{Sansone, A. Morandi, P. Dupplicato, E. Ungaro}

From Galeazzi Orthopaedic Institute, Milan, Italy

V. Sansone, MD, Professor of Orthopaedics

A. Morandi, MD, Orthopaedic Surgeon

Università degli Studi di Milano, Istituto Ortopedico Galeazzi, Via R Galeazzi 4, 20161 Milan, Italy.

P. Dupplicato, MD Orthopaedic Surgeon E. Ungaro, MD, Resident Istituto Clinico Humanitas IRCCS, Via Monzon 56, 20089 Rozzano, Italy.

Correspondence should be sent to Professor V. Sansone; e-mail: valerio.sansone@unimi.it

(C)2010 British Editorial Society of Bone and Joint Surgery doi:10.1302/0301-620X.92B6. $23171 \$ 2.00$

$J$ Bone Joint Surg [Br] 2010;92-B:807-10. Received 28 July 2009; Accepted after revision 16 February 2010

\author{
There are few reports of the surgical treatment for late stage Freiburg's disease with \\ flattening of the metatarsal head and osteoarthritis. We describe the results of the surgical \\ treatment of ten consecutive patients with advanced stage Freiburg's disease (Smillie's \\ stage V), using a technique that has not been published previously.
}

Freiburg's disease, or osteochondrosis of the epiphysis of a distal metatarsal, is seen most often in teenage girls. The second metatarsal head is affected in $68 \%$ of cases, and the third in $27 \% .^{1} \mathrm{~A}$ small number of cases are bilateral $(6.6 \%)$. Smillie $^{2}$ classified the disease as: stage I - epiphyseal fissuring; stage II - alteration of the contour of the articular surface; stage III sinking of the centre of the joint surface; stage IV - formation of a loose body; and stage V flattening of the metatarsal head and osteoarthritis. Conservative treatment is recommended initially for every stage and surgery is performed only if this fails. The management is usually conservative in the early disease (stages I and II), and operative in stages III, IV and V. Several operations have been described, but there is no consensus as to the best procedure, ${ }^{3}$ and authors have usually excluded stage $\mathrm{V}$ cases, when the joint is stiff and painful with impaired gait from their series. However, even in this late stage, if a conservative approach has failed surgery is indicated.

We have retrospectively examined a consecutive series of patients suffering from late stage Freiburg's disease, who did not respond to conservative treatment and underwent resectionarthroplasty and implantation of a temporary metal spacer. To our knowledge, this is the first series of stage V Freiburg's disease treated surgically and the operative technique has not been previously described.

\section{Patients and Methods}

Between October 2004 and June 2008 we treated ten consecutive patients who were suffering from metatarsalgia of the second (9) and third (1) metatarsal due to stage V Freiburg's disease and who had not responded to conservative treatment. There were nine women and one man, with a mean age of 46.2 years ( 24 to $67)$. The left foot was affected in seven cases and the right in three. Hallux valgus was present in five cases, metatarsophalangeal valgus deformity in four and interphalangeal valgism in one. A fixed hammer deformity of the second toe was present in one patient. In all cases, the associated pathology was symptomatic and received concomitant surgical treatment.

Operative technique. All patients are given preoperative antibiotic prophylaxis of $2 \mathrm{~g}$ of first generation cephalosporin. They are also prescribed low molecular weight heparin by bodyweight for 35 days after operation. The operation is performed under regional anaesthesia (lepobupivacaine hydrochloride), using a pneumatic tourniquet above the calf, inflated to $250 \mathrm{mmHg}$. A $4 \mathrm{~cm}$, slightly S-shaped, dorsal longitudinal incision is made, centred over the affected metatarsophalangeal joint.

After retraction of the extensor apparatus, a longitudinal capsulotomy is performed (Fig. 1 ). The collateral ligaments are released and any bony spurs removed from the metatarsal head. Approximately $2 \mathrm{~mm}$ to $3 \mathrm{~mm}$ of the base of the proximal phalanx are resected with an oscillating saw (Fig. 2). When the appropriate joint space has been created, the resected bone surface is made uniformly concave with a motorised burr, in order to accommodate the spacer which is convex on the phalangeal side and concave on the metatarsal aspect. The metal spacer (Button spacer, DePuy International Ltd., Leeds, United Kingdom) is tested for size, then implanted and fixed with a $1.2 \mathrm{~mm}$ Kirschner (K)-wire drilled firstly through the toe, through a hole in the centre of the spacer and onward into the metatarsal bone (Figs 3 and 4). This is followed by closure of the joint 


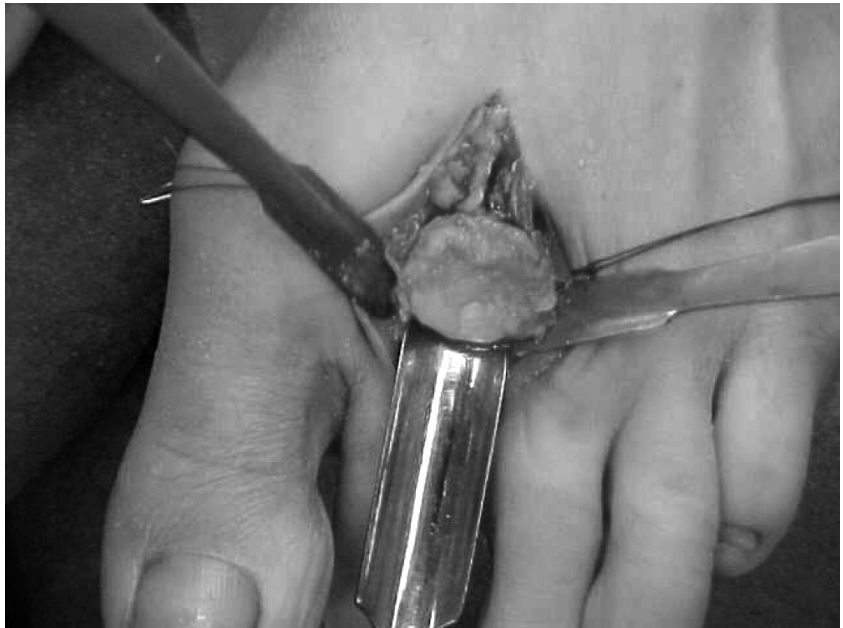

Fig. 1

Photograph showing a dorsal metatarsophalangeal capsulotomy and exposure of the deformed metatarsal head.

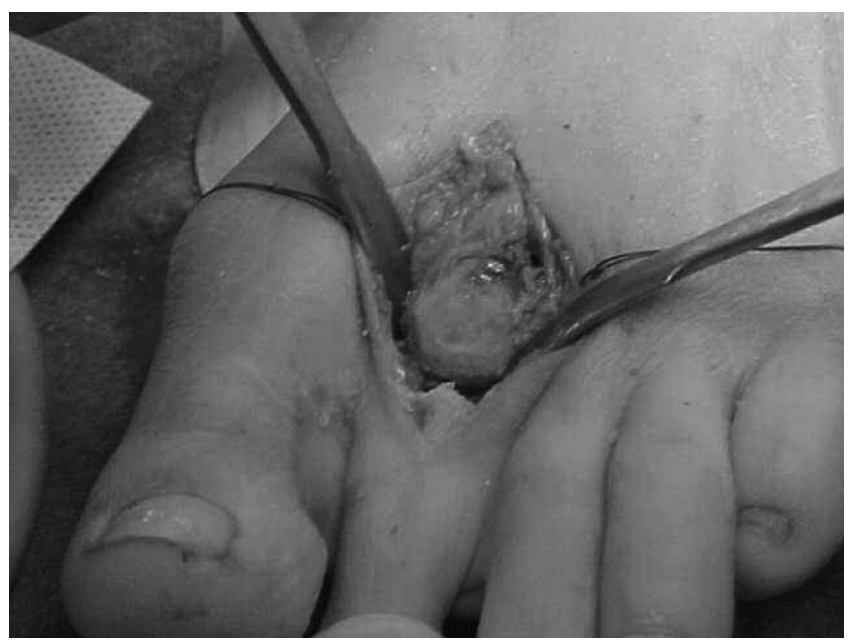

Fig. 2

Photograph showing a remodelled metatarsal head after removal of spurs and resection.

capsule, haemostasis and suturing. The mean tourniquet time was 20 minutes (17 to 20 ). All the patients are discharged the following day with a post-operative talus shoe which has a heel wedge to unload the forefoot. This shoe is worn for three weeks post-operatively. Full weight-bearing is allowed immediately, unless there is pain. The Kwire is removed after three weeks and the patients are instructed to manipulate the toe gently to regain movement.

The spacer is removed as a day case six months after the initial operation under regional anaesthesia and through the same incision. Care is taken not to damage the extensor apparatus and to ensure an accurate reconstruction of the metatarsophalangeal capsule. After operation, the patients

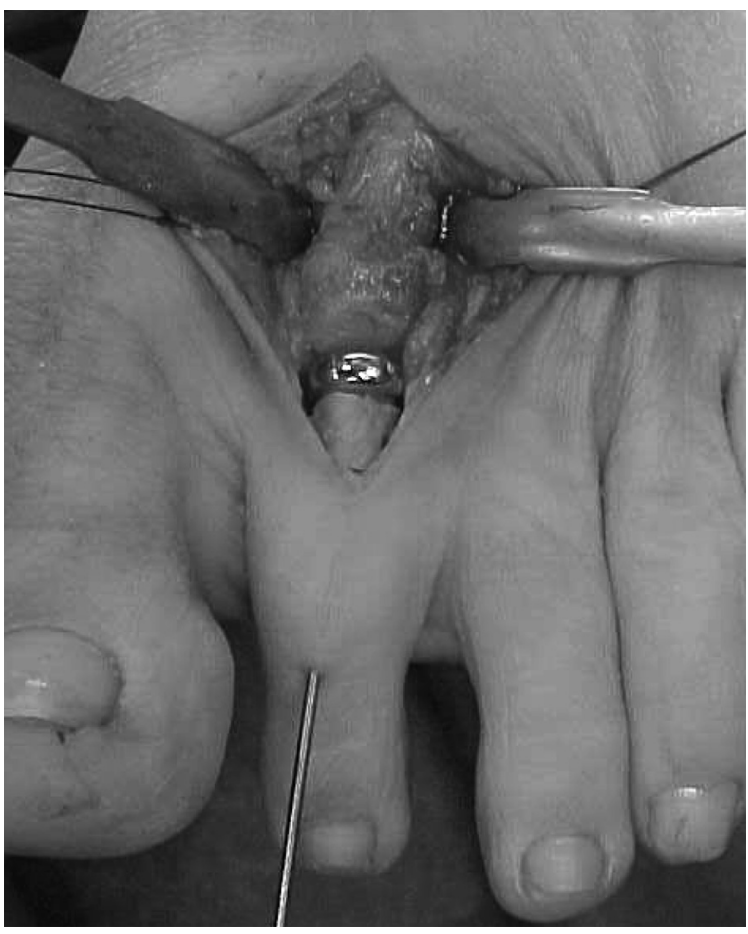

Fig. 3

Photograph showing the spacer has been implanted and stabilised with a Kirschner wire.

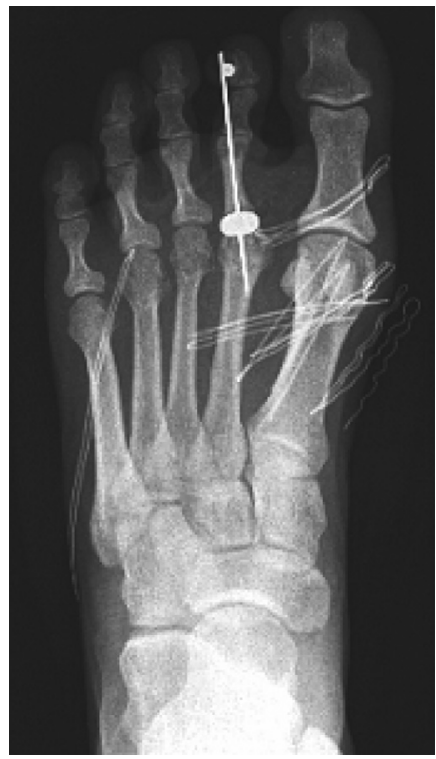

Fig. 4

Post-operative radiograph showing the results of cheilectomy, resection of the base of the proximal phalanx, and implantation of the spacer.

wear a talus shoe for seven days. They are instructed to start moving the toe manually as soon as possible.

Pre-operative weight-bearing radiographs were taken in all cases (Fig. 5). For the first 12 months the patients under- 


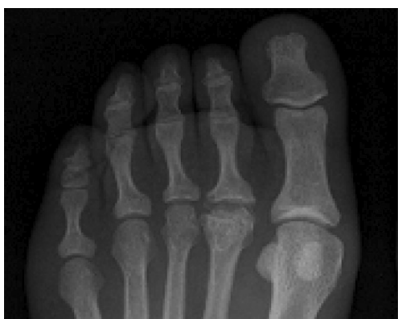

Fig. 5

Pre-operative weight-bearing radiograph showing advanced degenerative changes in the left second metatarsophalangeal joint.

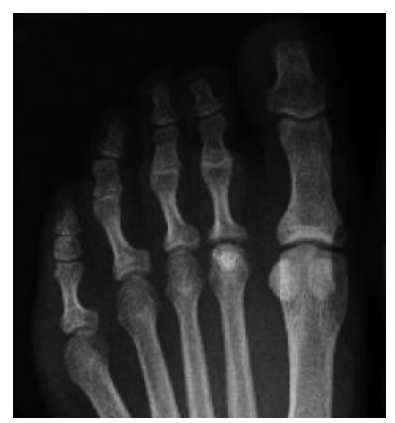

Fig. 6

Follow-up radiograph taken after the spacer has been removed and the joint space re-established. The shortening of the proximal phalanx is negligible.

went periodic clinical and radiological review (Fig. 6), which were subsequently carried out annually. The mean follow-up was for 19 months (12 to 36) after removal of the spacer and the clinical results were evaluated preoperatively and at follow-up according to the 100 point AOFAS (American Orthopaedic Foot and Ankle Society) lesser metatarsophalangeal-interphalangeal scale. ${ }^{4}$

\section{Results}

The pre-operative and post-operative scores, are shown in Table I where 90 or more points was considered as excellent; 80 to 89 , good; 70 to 79 , fair; and 69 points or less as poor. Pre-operatively, all patients had pain and limitation of metatarsophalangeal movement. No patient was lost to follow-up. The mean scores were 39.0 (23 to 45) preoperatively and 83.9 (53 to 97) post-operatively. Subjectively, three patients were very satisfied with the outcome with almost complete resolution of symptoms and an excellent recovery of joint function; six were much improved, with only occasional pain which did not limit their daily activities and one was dissatisfied. The mean results for range of movement of the metatarsophalangeal joint were
Table I. Pre- and post-operative American Orthopaedic Foot and Ankle Society scores

\begin{tabular}{cccll}
\hline \multicolumn{2}{l}{ Patients } & Gender (yrs) & $\begin{array}{l}\text { Pre-operative Post-operative } \\
\text { score }\end{array}$ & $\begin{array}{l}\text { Pge } \\
\text { score }\end{array}$ \\
\hline 1 & F & 37 & 38 & 85 \\
2 & F & 53 & 40 & 85 \\
3 & F & 56 & 39 & 80 \\
4 & F & 37 & 40 & 87 \\
5 & M & 24 & 42 & 90 \\
6 & F & 39 & 45 & 95 \\
7 & F & 53 & 38 & 82 \\
8 & F & 38 & 43 & 97 \\
9 & F & 58 & 42 & 85 \\
10 & F & 67 & 23 & 52 \\
\hline
\end{tabular}

Table II. Pre- and post-operative American Orthopaedic Foot and Ankle Society (AOFAS) scores for range of movement

\begin{tabular}{lll}
\hline & \multicolumn{2}{l}{ Number of patients } \\
\cline { 2 - 3 } AOFAS range of movement $\left({ }^{\circ}\right)$ & Pre-operative & Post-operative \\
\hline Normal or minimal reduction $>75$ & 0 & 5 \\
Moderate reduction 30 to 74 & 0 & 3 \\
Severe reduction $<30$ & 10 & 2 \\
\hline
\end{tabular}

$15^{\circ}$ pre-operatively, and $75^{\circ}$ post-operatively (Table II). We did not observe any complications.

\section{Discussion}

The operative treatment of late stage Freiburg's disease aims to relieve pain, restore metatarsophalangeal function and re-establish the correct distribution of body weight. ${ }^{5}$ Procedures employed for advanced disease include excision of the metatarsal head and/or base of the proximal phalanx, shortening of the metatarsal, intra-articular or extraarticular dorsiflexion osteotomy of the metatarsal head, interposition arthroplasty with a biological or silicon spacer and osteochondral plug transplantation. . $^{5-13}$

Dorsal wedge osteotomy, whether intra- ${ }^{7,8}$ or extraarticular, ${ }^{9}$ is a standard technique with good and easily reproduced results. However, the procedure has disadvantages. Shortening of the second ray changes the weight distribution and frequently leads to transfer metatarsalgia. ${ }^{10} \mathrm{Chao}$ et $\mathrm{al}^{9}$ described AOFAS scores comparable with our results after a longer follow-up of 40 months, with four excellent, seven good, and two poor or fair. However, their technique involved a mean shortening of the metatarsal of $2.1 \mathrm{~mm}$, and one patient suffered transfer metatarsalgia as a result. Furthermore, if the position of the articular surface is modified by dorsiflexion, dorsal metatarsophalangeal joint movement is reduced. Kinnard and Lirette ${ }^{7}$ found a mean loss of $15^{\circ}$ of plantar flexion and $10^{\circ}$ of extension, and Chao et $\mathrm{al}^{9}$ a mean reduction of $15^{\circ}$ of flexion and $8^{\circ}$ of extension. These results compare favourably with our observations, although direct comparison is difficult as they are not presented in the three class AOFAS format for recording range of movement. 
Cracchiolo et $\mathrm{al}^{11}$ observed that interpositional arthroplasty with a silicon spacer had not proved totally satisfactory, as the operated metatarsal often cannot support the mechanical stresses imposed on it. Many of the patients are young, with high functional demands which risk displacing the implant. ${ }^{11}$ They also reported cases of synovitis caused by the foreign body, and transfer metatarsalgia. At a mean follow-up of 37 months, their rate of failure was $12 \%$.

The interposition of biological material, either the flexor or extensor tendons, or joint capsule has been used with some success. The technique has been refined to the extent that some authors have proposed undertaking the procedure arthroscopically. ${ }^{12}$ Very good results are reported by these authors in terms of pain relief, although recovery of joint movement is less satisfying, probably because the technique does not guarantee the re-establishment of an adequate joint space.

Comparison with arthroscopic osteochondral plug transplantation is more complex. This technique could be an interesting solution, but to date, only very few preliminary reports are available. ${ }^{13}$ The operation is limited by the technical demands of operating arthroscopically in a small joint space which is further reduced by disease. Also, as the graft is harvested from the knee, there may be complications at the donor site.

Patients with late stage Freiburg's disease have a limited range of movement due to deformity of the articular surfaces and a significantly reduced joint space. In our opinion, movement cannot be restored by undertaking a simple debridement and cheilectomy in these cases. Our technique involves minimal resection of the base of the proximal phalanx to recreate an adequate joint space and restore a more congruent articulating surface. The temporary spacer maintains the distance, thereby allowing the formation and growth of a layer of reparative tissue to cushion the joint. From these observations and histological studies after abrasion treatment for full-thickness chondral lesions in the knee, ${ }^{14}$ it can be reasonably supposed that the tissue layer we observed is made of resilient fibro-cartilage. In our experience, after six months, this is sufficiently thick and resilient for the spacer to be removed. In the last few years we have used metal spacers in the treatment of hallux rigidus with good results, due to their excellent tolerance, good mechanical properties and relatively low cost. To our knowledge, a temporary metal spacer has not been described previously for treatment of late stage Freiburg's disease and we consider that it has several advantages over the techniques previously described. The length of the metatarsal remains substantially unchanged which avoids transfer metatarsalgia. The risk of rejection or breakage of the implant is avoided, and it guarantees an adequate space while it remains in place. The surgical technique for implantation and removal of the spacer is easy and requires minimal instrumentation. The longitudinal dorsal approach gives an excellent view of both sides of the joint, which enables the surgeon to re-model the metatarsal head and prepare the phalanx. The procedure is minimally invasive and only requires the resection of a small amount of bone, which allows immediate weight-bearing and early return of function if a talus shoe is used post-operatively.

A possible concern with this technique is shortening of the toe, however, any shortening we observed was minimal (Fig. 6), and caused no functional or aesthetic problems. Although we observed no significant reduction in the joint space in the follow-up radiographs, we cannot exclude the possibility of deterioration over time. A longer follow-up is needed to establish whether our good clinical and radiological results are maintained. Another disadvantage of our technique is that a second operation, albeit minor, is required to remove the spacer. If reliable bioreabsorbable spacers become available, a second operation will no longer be necessary. Our technique has some minor limitations but we feel that the good results make it a valid alternative to existing operations.

No benefits in any form have been received or will be received from a commer cial party related directly or indirectly to the subject of this article.

\section{References}

1. Shih AT, Quint RE, Armstrong DG, Nixon BP. Treatment of Freiberg's infraction with the titanium hemi-implant. J Am Podiatr Med Assoc 2004;94:590-3.

2. Smillie IS. Freiberg's infraction (Kohler's second disease). J Bone Joint Surg [Br] 1957;39-B:580.

3. Katcherjan DA. Treatment of Freiberg's disease. Orthop Clin North Am 1994;25:69-81.

4. Kitaoka HB, Alexander IJ, Adelaar RS, et al. Clinical rating systems for the anklehindfoot, midfoot, hallux and lesser toes. Foot Ankle Int 1994;15:349-53.

5. Carmont MR, Rees RJ, Blundell CM. Current concepts review: Freiberg's disease. Foot Ankle Int 2009;30:167-76.

6. Smith TW, Stanley D, Rowley DI. Treatment of Freiberg's disease: a new operative technique. J Bone Joint Surg [Br] 1991;73-B:129-30.

7. Kinnard P, Lirette R. Freibeurg's disease and dorsiflexion osteotomy. J Bone Joint Surg [Br] 1991;73-B:864-5.

8. Gauthier G, Elbaz R. Freiberg's infraction: a subchondral bone fatigue fracture: a new surgical treatment. Clin Orthop 1979;142:93-5.

9. Chao KH, Lee CH, Lin LC. Surgery for symptomatic Freibeg's disease: extraarticular dorsal closing-wedge osteotomy in 13 patients followed for 2-4 years. Acta Orthop Scand 1999;70:483-6

10. Carro LP, Golano P, Farinas $\mathbf{0}$, Cerezal L, Abad J. Arthroscopic Keller technique for Freiberg disease. Arthroscopy 2004;20:60-3.

11. Cracchiolo A 3rd, Kitaoka HB, Leventen EO. Silicone implant arthroplasty for second metatarsophalangeal joint disorders with and without hallux valgus deformities. Foot Ankle 1988;9:10-18.

12. Lui TH. Arthroscopic interpositional arthroplasty for Freiberg's disease. Knee Surg Sports Traumatol Arthrosc 2007;15:555-9.

13. Miyamoto W, Takao M, Uchio Y, Kono T, Ochi M. Late-stage Freiberg disease treated by osteochondral plug transplantation: a case series. Foot Ankle Int 2008;29:950-5

14. Johnson LL. Arthroscopic abrasion arthroplasty: a review. Clin Orthop 2001;391(Suppl):306-17 